\title{
Phonemic Testing
}

A. G. EPSTEIN (Speech Theropist)

Tamperdalsvej II. Arhus. Denmark

Articulation testing of children with so-called "articulatory disorders" is carried out by almost every speech therapist. In logopedic literature "articulatory disorders" are divided into "omissions, distortions and substitutions". I would say that firstly, the expression "articulation testing" is not very good unless one is speaking about dysarthria, and secondly, that the division of different kinds of errors into "omissions, distortions and substitutions" is not a satisfactory classification from my present point of view. In the following article I shall attempt to elucidate this viewpoint and afterwards demonstrate how by testing the children, one can get an exact figure which measures their performance in the test. This figure can be entered into a system of coordinates with the figure of the test performance as the ordinate and time as the abscissa.

To master a language one must: (a) know its system of phonemes and use it in the correct way, (b) possess a sufficiently large vocabulary in that language, (c) have an adequate knowledge of its grammar. Instead of "articulation tests" which test speech, one should speak of testing the phonemic system i.e. a testing of language and not speech.

In this frame of reference, "omissions" mean "the use of too few phonemes", for example, where you get 'p' for 'pr' ('pice' for 'price') ' $s$ ' for 'sn' ('sow' for 'snow') or nothing for ' $r$ ' ('ice' for 'rice'). 'Substitutions" mean "the wrong use of phonemes", for example, ' $t$ ' substituted for ' $c$ ' ('tat' for 'cat'). If the substitution of ' $t$ ' for ' $c$ ' remains consistent, one has an omission at the same time, as the ' $\mathrm{c}$ ' phoneme is not used at all. Such a case could be termed an "omission-substitution".

"Distortion" can be considered as a real speech defect, a faulty articulation of a phoneme. The distortion of an ' $\mathrm{s}$ ' for instance, is understood as an ' $\mathrm{s}$ ' and does not reduce the number of phonemes used in a language. Thus while distortions are speech defects, omissions and substitutions are defects in linguistic expression i.e. they are language defects. To master a language it is necessary to use its units of expression correctly. There are a certain limited number of single consonants, vowels and consonant groups i.e. a limited number of linguistic units of expression. To master the total number of expressive units means to master the vocabulary in its correct normal form, which is immediately understood by anyone speaking that language. In the English language the total number of expressive units is between $100-200$. This modest sum includes single consonants, in initial, medial and final positions, plus all conjunctive consonant groups (i.e. appearing within one syllable). The Danish test of the Arhus Institute has 225 units, as some disjunctive consonant groups, appearing in different but neighbouring syllables, act conjunctively, e.g. the word "cocktail", s are included. These disjunctive groups may however be omitted in a test examining the linguistic expressive units.

The person to be tested is asked to repeat these expressive units, which have been put into words. The examiner says the word once. Note is taken of each response and an estimate is made of the correct responses. If at the first testing, 100 answers of the 225 units are correct, the degree of mastery of the expressive units of the language is stated as $100 / 225$. At a test about one or two months later, the person may give incorrect responses where previously they were correct. For instance, 5 units which were correct at the first test may now be incorrect; and at the same time ten units, previously incorrect, may now be correct. Thus he will have advanced five points $(10-5)$ on his previous score i.e. he is now scoring $105 / 225$.

On a sheet of graph paper, it is possible to make a system of co-ordinates, as previously described, counting one unit per millimeter on the ordinate, and one month per ten millimeters on the abscissa. Each millimeter line represents a date divisable by 3 . In this way a curve showing progress in the acquisition of linguistic expressive units will be obtained.

It might also be useful to find the relationship be.tween the increase (positive change) and decrease (negative change) on a certain date of testing i.e. how great is the vacillation. This can be established in the following way:

A horizontal line, or zero line, 30-40 millimeters from the lowest edge of the paper is drawn. This is used as the abscissa. A point is made on the zero line, vertically under the testing date. From this point a line is drawn upwards representing in millimeters the number of nerv correct answers. This line is continued under the zero line for a length corresponding in millimeters to the number of incorrect answers which were formerly correct. The difference in length of the two lines thus drawn above and below the zero line expresses the gain or loss on the date in question. 


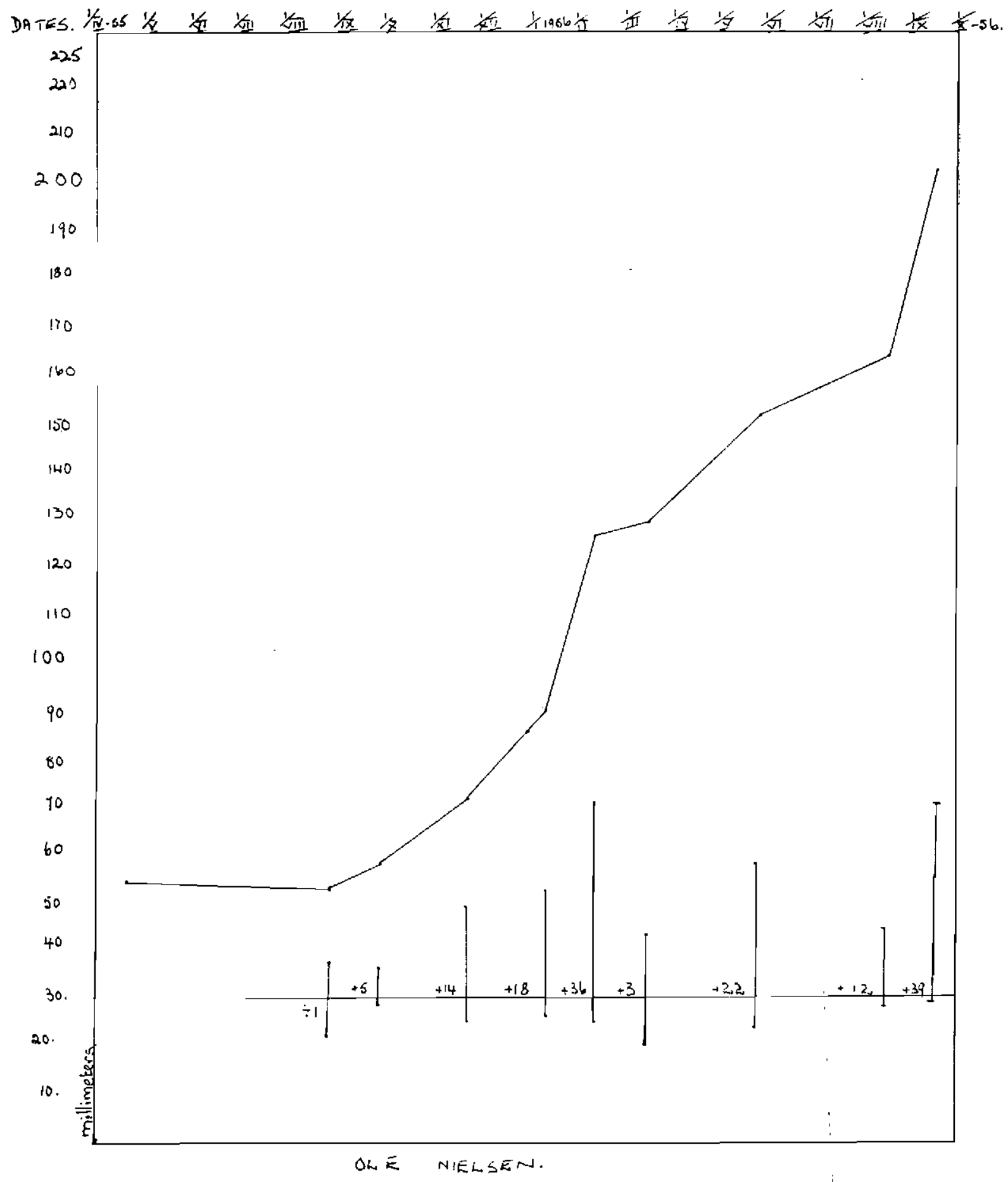

